\title{
Probleme wort- und satzgrammatischer Terminologie aus der Sicht der Schulgrammatik
}

\section{Die Ursachen für die Deskriptorenvielfalt}

Jede Form des Deutschunterrichts braucht - mögen auch die didaktischen und methodischen Akzente innerhalb des Lernbereichs "Reflexion über sprachen unterschiedlich gesetzt sein - eine Beschreibungssprache, um sprache als Kommunikationsmittel und als Kommunikationssystem durchschaubar, verstehbar und metasprachlich verfügbar $\mathrm{zu}$ machen.

Daher haben die Didaktiker des Faches Deutsch sowie die Autoren von Sprachbüchern die Beschreibungsmodelle und Deskriptoren zur Verfügung zu stellen, die eine Beschreibung der sprache in ihrer kommunikativen Funktion und als geordnetes system sprachlicher zeichen ermöglichen.

Da die Sprachwissenschaft mehrere sehr unterschiedliche Beschreibungsmodelle anbietet, muste sich der Didaktiker, und insbesondere der sprachbuchautor, entweder für ein hier und da aus didaktischen Gründen verändertes Beschreibungsmodell entscheiden, oder er entnahm den verschiedenen Beschreibungsmodellen ihm geeignet erscheinende Elemente, so daß es in den Sprachbüchern häufig $z u$ einer Mischung von Deskriptoren aus unterschiedlichen Beschreibungsmodellen $\mathrm{kam}$. Es entstand als schulgrammatik jeweils eine sog. "Mischgrammatik".

Da sehr viele sprachbuchautoren ihren Werken Mischgrammatiken zugrunde legten und die einzelnen Beschreibungsmodelle in unterschiedlicher weise favorisierten, ergab sich der für die Schulgrammatik insgesamt problematische zustand, daß eine Fülle unterschiedlicher grammatischer Deskriptoren und mehrere unterschiedliche Beschreibungsmodelle Lehrer und Schüler verwirrten.

Die Analyse der verwendeten Beschreibungsmodelle und Deskriptoren sowie der graphischen Darstellungen deutscher sätze läbt erkennen, das die folgenden Beschreibungsmodelle Pate gestanden haben: 
- die traditionelle Grammatik, deren Deskriptoren sich an der überlieferten lateinischen Terminologie orientieren;

- die Konstituentenstrukturgrammatik (KStG);

- die generative Transformationsgrammatik (GTG);

- die Dependenzgrammatik;

- die inhaltbezogene Grammatik.

II. Der Wunsch nach einer einheitlichen grammatischen Terminologie

Angesichts der geschilderten Vielfalt an grammatischen Termini, Beschreibungsmodellen und graphischen Darstellungen ist es nur zu verständlich, daß der wunsch nach einer einheitlichen Terminologie zumindest im Bereich der Schulgrammatik laut wurde.

So wünschen sich die schulen und hier insbesondere die Gymnasien, daß die Schulbücher der sprachlichen Fächer, also Deutsch sowie alte und neue Fremdsprachen, eine einheitliche oder zumindest kompatible Terminologie aufweisen.

Auch ist es wünschenswert, daß schüler, die in ihrer schullaufbahn von einer schulform zu einer anderen wechseln, nicht auch die grammatische Terminologie wechseln müssen.

Ferner wäre es für schulbuchverlage und schulbuchautoren eine Erleichterung ihrer Arbeit, könnten sie eine Terminologie verwenden, die in den schulen aller Bundesländer anerkannt wäre, so daß sie nicht auf landesspezifische Forderungen und Besonderheiten Rücksicht nehmen müßten.

Und auch für die Universitäten dürfte es kein Nachteil sein, wenn die studenten von ihrer schulbildung her über ein angemessenes grammatisches Grundwissen mit einer einheitlichen, im deutschen sprachraum verstehbaren Terminologie verfügten (und dies unbeschadet der wissenschaftlichen Notwendigkeit, daß neue Sehweisen von sprache und neue Beschreibungsmodelle ihre eigenen modellkonformen Deskriptoren entwickeln und auch entwickeln sollen).

Der erkennbare Wunsch nach einer Vereinheitlichung der grammatischen Terminologie impliziert aber - und das mus deutlich ge- 
sehen werden - den wunsch nach einem bestimmten Beschreibungsmodell, nämlich dem, für dessen Deskriptoren man sich entscheidet. Denn grammatische Termini sind als Deskriptoren sprachlicher Phänomene in der Regel Elemente eines umfassenden, nach Möglichkeit konsistenten Beschreibungsmodells, das die Teile eines komplexen Ganzen sowie deren Relationen erfabt, bezeichnet und verständich macht.

Wer also den Versuch macht, die Terminologie der schulgrammatik zu vereinheitlichen, müßte auch den Mut haben, offen zu sagen, welches Beschreibungsmodell - und dies ist das entscheidende problem im Bereich der satzgrammatik - einer einheitlichen Schulgrammatik zugrunde gelegt werden soll.

III. Versuche, die Terminologie der Schulgrammatik zu vereinheitlichen

Aufgrund der immer deutlicher artikulierten Forderung, die grammatische Terminologie der schulsprachen zu vereinheitlichen, sind in den letzten fünf Jahren zwei umfassende Vorschläge zur Vereinheitlichung der Terminologie gemacht worden:

- Basisliste grammatischer Fachausdrücke,

Arbeitskreis "Abstimmung der grammatischen Terminologie",

Landesstelle für Erziehung und Unterricht, Stuttgart, Juni 1979;

- Verzeichnis grundlegender grammatischer Fachausdrücke,

Sekretariat der ständigen Konferenz der Kultusminister der Länder, Anlage VI z. NS. AK. 26.2.1982, Bonn (KMK-Liste).

Das Problem der uneinheitlichen, verwirrenden, teilweise auch widersprüchlichen Terminologie ist jedoch durch die vorgelegten Vereinheitlichungsvorschläge noch nicht gelöst worden. Einmal ist die zeit seit dem Erscheinen dieser Vorschläge zu kurz, als daB sie bereits in Sprachbüchern oder Richtlinien ihren deutlichen Niederschlag gefunden haben könnten. Zum anderen sind die Deskriptoren und Beschreibungsmodelle, für die sich die einzelnen Schulbuchautoren entschieden haben, insgesamt $z u$ unterschiedlich, als daß sich die Autoren von heute auf morgen wider- 
spruchslos der einen oder anderen vorgeschlagenen Terminologie und dem damit verbundenen Beschreibungsmodell unterwerfen wïrden.

Wenngleich das Erscheinen der KMK-Liste auch von verschiedenen Seiten begrüBt worden ist, darf nicht übersehen werden, daB es auch kritisiert worden ist, weil es, wie gesagt wurde, die vielfalt der vorhandenen Beschreibungsmodelle zu beschneiden und die schulgrammatische Terminologie auf administrativem wege zu regeln versuche.

Die gegenwärtige situation der schulgrammatik und ihrer Terminologie ist also dadurch gekennzeichnet, daB einerseits nicht unumstrittene vorschläge zur vereinheitlichung zwar vorliegen, ohne daß es tatsächlich zu der von vielen gewünschten vereinheitlichung gekommen wäre, und andererseits die versuche, die Terminologie der schulgrammatik zu vereinheitlichen, heftig kritisiert werden, weil sie die wissenschaftliche Diskussion um die Beschreibungsmodelle abschnürten sowie Lehrer und schulbuchautoren einem administrativen zwang unterwürfen.

Damit wirft die gegenwärtige situation aufgrund der geschilderten Kontroverse einige grundsätzliche Fragen auf, die das Verhältnis von Wissenschaft und Didaktik betreffen:

- MuB die Didaktik die Diskussion um die Beschreibungsmodelle und die Deskriptoren offen halten, nur weil diese Diskussion in der wissenschaft offen ist?

- Wäre es nicht auch vertretbar, daß die Fachdidaktik um der Schule und der schüler willen trotz der offenen wissenschaftlichen Diskussion eine Entscheidung in Richtung einer terminologischen Vereinheitlichung trifft, damit die schüler mit einem einzigen Beschreibungssystem und dessen Terminologie vertraut gemacht werden und damit arbeiten können? Denn didaktisch unbestritten ist, das schüler der Klassen 3 bis 13 in der Regel nicht mehrere unterschiedliche Beschreibungsmodelle und deren Deskriptoren nebeneinander verwenden sollen.

- Ist es den sprachwissenschaftlern des deutschen sprachraums nicht möglich, sich auf ein bestimmtes Beschreibungsmodell und 
dessen Deskriptoren zu einigen, um es der Fachdidaktik als ein Basisinstrument der schulischen sprachanalyse zu empfehlen?

- Oder könnten nicht zumindest die Didaktiker der einzelnen Schulfächer - etwa im Rahmen ihrer Fachverbände - versuchen, sich auf eine für den schulischen Gebrauch bestimmte vereinheitlichte Terminologie zu verständigen?

Da zur zeit wenig Aussicht besteht, dab sowohl die sprachwissenschaftler als auch die Fachdidaktiker untereinander und miteinander $z u$ einem tragfähigen Konsens hinsichtlich der grammatischen Terminologie gelangen, soll hier der versuch gemacht werden, auf dem Markt vorhandene Sprachbücher vor dem Hintergrund der vorschläge der KMK-Liste miteinander zu vergleichen, um das Maß der schon bestehenden Ubereinstimmung in der verwendeten wort- und satzgrammatischen Terminologie zu ermitteln und für die Phänomene der Wort- und Satzgrammatik, bei denen der Dissens unter den Schulbuchautoren offenkundig ist, Vorschläge zur terminologischen vereinheitlichung zu machen.

Es sollen also insbesondere die gegenwärtig vorhandenen Problemfälle in der wort- und satzgrammatischen Terminologie der schulgrammatik dargestellt und Vorschläge zur Bezeichnung kontrovers deskribierter Phänomene gemacht werden.

\section{Probleme der wortgrammatischen Terminologie}

Vergleicht man Sprachbücher, die z.zt. auf dem schulbuchmarkt sind, im Blick auf die wortartenbezeichnungen miteinander ${ }^{l}$, so stellt man in diesem terminologischen Bereich weitgehend Ubereinstimmung fest. Fast iberall finden sich die Wortartenbezeichnungen, die der traditionellen Grammatik zuzurechnen sind und auf die lateinische Terminologie zurückgehen. Bezeichnungsvarianten, die aus Elementen des Deutschen gebildet sind, kommen äuBerst selten in Sprachbüchern ab Klasse 5 vor.

Da die heute im Deutschen verwendeten Wortartenbezeichnungen der sog. "traditionellen Grammatik" auf Lexemen basieren, die auch den Wortartenbezeichnungen zugrunde liegen, die sowohl in sprachwissenschaftlichen Darstellungen des Englischen, Französi- 
schen und Spanischen als auch in schulbüchern dieser sprachen verwendet werden, stehen für den Bereich der Wortarten gleichsam international verstehbare Termini zur verfügung. ${ }^{2}$

Die terminologischen Probleme im Bereich der wortarten sind relativ gering, im einzelnen handelt es sich um folgende probleme: Der Sprachgebrauch der Altsprachler, wonach der Terminus "Nomen" Oberbegriff $z u$ den Begriffen "Substantiv", "Pronomen", "Adjektiv" und "Numerale" ist, sollte zugunsten des Denotats 'substantiv', 'Hauptwort' aufgegeben werden. De facto liegt in fast allen herangezogenen sprachbüchern eine Begrenzung auf das eben genannte Denotat vor, und dasselbe gilt für die Denotate der englischen und französischen Korrespondenztermini "noun" und "nom". Daraus ergibt sich, daB die Auffassung, wie sie sich in der KMK-Liste ablesen läßt: "Substantive sind eine Untergruppe der Nomina" ${ }^{3}$, aufgegeben werden sollte. Tatsächlich kommt der Terminus "substantiv" in vielen sprachbüchern als synonym zu dem Terminus "Nomen" vor. Zu folgen wäre allerdings dem Vorschlag der KMK-Liste, wonach der Terminus "Nomen" dem Terminus "Substantiv" vorgezogen werden sollte, da er besser $z u$ den verwandten Termini "Nominalgruppe", "Pronomen" und "Prädikatsnomen" paßt.

Demgegeniber sollte der Terminus "Pronomen" ausschlieblich als Oberbegriff $z u$ den verschiedenen Unterbegriffen, die mit dem Grundwort "-pronomen" gebildet werden, verwendung finden und nicht als Synonym zu dem Unterbegriff "Personalpronomen".

Da sowohl aus sprachwissenschaftlichen als auch aus didaktischen Gründen wichtig ist, klar zwischen wortartentermini und Satzgliedtermini zu unterscheiden, sollte - entsprechend dem Vor schlag der KMK-Liste - der Terminus "Adverb" ausschlieblich als Wortartenterminus für indeklinable wörter wie sehr, gestern, heute und nicht für adverbial gebrauchte Adjektive verwendet werden.

Trotz der drei dargestellten terminologischen probleme kann aber festgestellt werden, daB im Bereich der Wortartentermini unter den Schulbuchautoren weitgehend Ubereinstimmung besteht. Das MaB der vorhandenen Ubereinstimmung zeigt die folgende Ubersicht: 

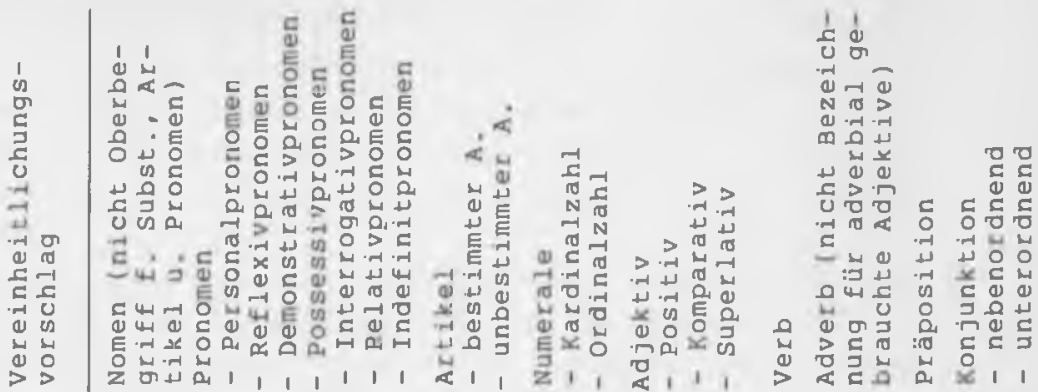

\begin{tabular}{|c|c|c|c|c|c|c|c|c|c|}
\hline $\begin{array}{l}\overrightarrow{0} \\
\stackrel{1}{-1} \\
0 \\
0 \\
\vec{\Delta} \\
\dot{\Delta}\end{array}$ & 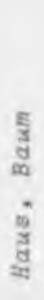 & 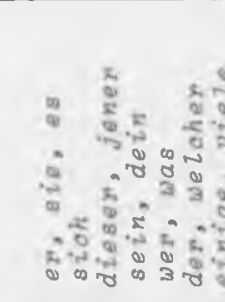 & 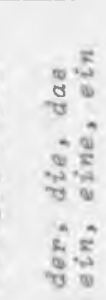 & 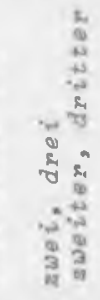 & 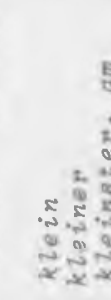 & 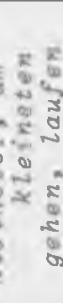 & $\begin{array}{l}5 \\
0 \\
0 \\
0 \\
0 \\
0 \\
0 \\
5 \\
0 \\
0 \\
0\end{array}$ & 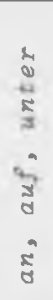 & 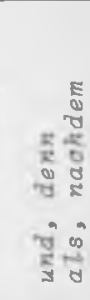 \\
\hline 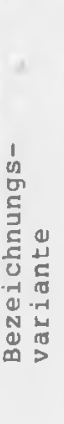 & $\begin{array}{l}\text { H } \\
0 \\
3 \\
3 \\
0 \\
0 \\
3 \\
0\end{array}$ & 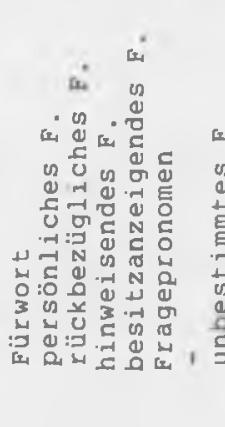 & 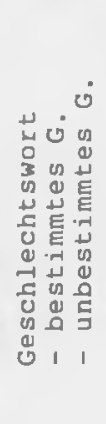 & 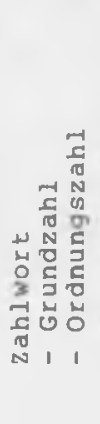 & 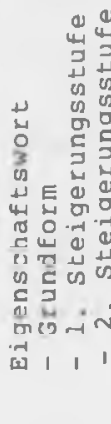 & 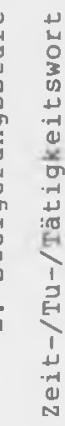 & 1 & 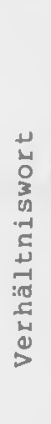 & 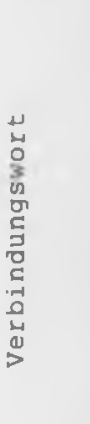 \\
\hline 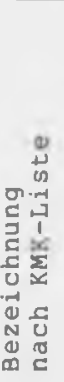 & 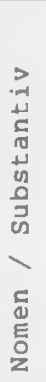 & 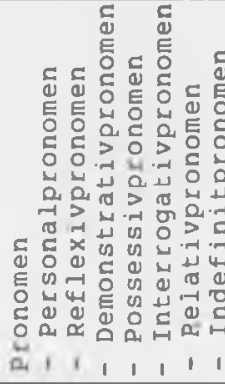 & 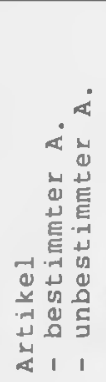 & 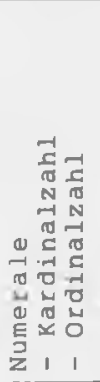 & 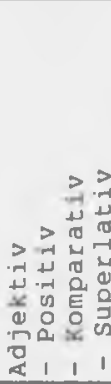 & $\begin{array}{l}\text { 음 } \\
\stackrel{0}{>}\end{array}$ & 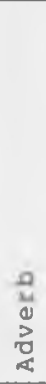 & 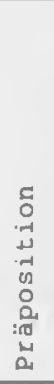 & 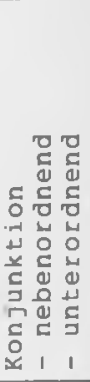 \\
\hline
\end{tabular}


Im Blick auf die Termini der Wortbildungslehre ergeben sich jedoch größere Divergenzen. So gibt es zu dem Terminus "stamm", wie er sich in der KMK-Liste findet, die Bezeichnungsvarianten "Wortkern", "Wortstamm", "Lexem", "stamm-Morphem" und "Stammwort". Im Gegensatz zu dem Vorschlag der KMK-Liste dürften die beiden Termini "Wortstamm" und "Lexem" die sachlich besten sein.

In der KMK-Liste vermiBt man einen Terminus wie "Endung" oder "Morphem", ein Terminus, der bei der Analyse von Nominal- und Verbalformen häufig benötigt wird. Beide genannten Termini scheinen geeignet, jeweils als Grundwort der folgenden Unterbegriffe zu fungieren:

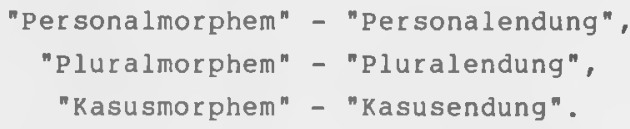

In Ubereinstimmung mit der KMK-Liste sollte man dem falschen Gebrauch des Terminus "Vorsilbe" bzw. "Nachsilbe" als Synonym für den Terminus "Präfix" bzw. "Suffix" wehren.

Im Blick auf Termini der Wortbildungslehre sollte die KMK-Liste noch um zwei Termini ergänzt werden: nämlich um den Terminus "Kompositum" (vgl. engl. compound, frz. le nom composé) an stelle der Bezeichnung "zusammengesetztes Wort" und um den Terminus "Simplex" mit dem Denotat 'nicht-zusammengesetztes Wort'. 


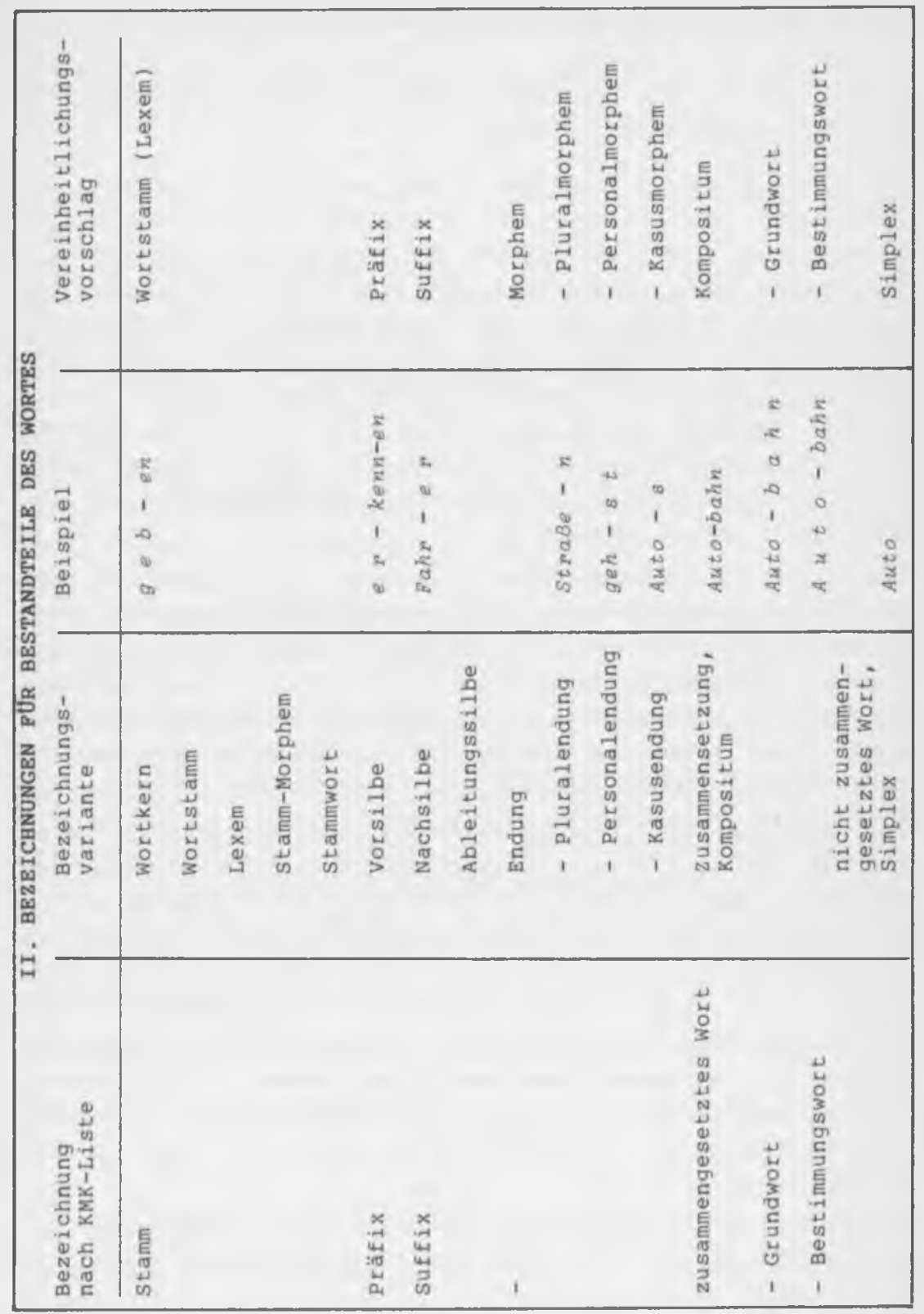


V. Probleme der satzgrammatischen Terminologie

Im Bereich der Satzgrammatik läßt sich unter den schulbuchautoren ein weitaus größerer Dissens feststellen, als dies im Bereich der Wortgrammatik der Fall ist.

Die größte Divergenz der Termini liegt bei den Bezeichnungen der Satzglieder vor. Die einzelnen Sprachbücher unterscheiden sich insbesondere in der Bezeichnung für die syntaktische Funktion des finiten Verbs eines Satzes. Die eine Gruppe der Autoren bezeichnet dieses Satzglied - wie die KMK-Liste - als "Prädikat" 5 , andere Autoren verwenden jedoch dafür die Termini "Verbalteil", "Satzkern" und "Prädikatskern"6. Hinzu kommt, daß diejenigen, die den Terminus "Prädikatskern" verwenden, auch den Terminus "Prädikat" benutzen, allerdings mit dem Denotat 'Satzaussage, die alle verschiebbaren, also selbständigen satzglieder mit Ausnahme des subjekts umfabt'.

Die beschriebene Divergenz beruht darauf, daß die genannten Termini im Rahmen unterschiedlicher Beschreibungsmodelle vorkommen. Wer den Terminus "Prädikat" mit dem Denotat 'verbalteil des Satzes' verwendet, beschreibt den Satz in der Regel atomistisch, d.h. er erfabt die einzelnen verschiebbaren satzglieder als Elemente einer kette von satzgliedern, damit wird der satz im wesentlichen in seiner oberflächenstruktur erfaßt.

Die Verwendung des Terminus "Prädikat" mit dem Denotat 'Satzaussage, die alle verschiebbaren satzglieder mit Ausnahme des subjekts umfabt' läвt darauf schlieben, daß der satz in seinen Makrosegmenten sowie nach den diesen zugeordneten verschiebbaren Teilelementen erfaBt werden soll, wobei nicht nur die oberflächenstruktur, sondern auch die Tiefenstruktur des satzes in den Blick kommt. Die eben beschriebene sehweise hat zur Folge, daß der Satz als Verknüpfung der Makrosegmente "Subjekt" und "Prädikat" interpretiert wird, wobei der Prädikatskern, die objekte und die Adverbialbestimmungen als Elemente des Prädikats verstanden werden.

Die zuletzt beschriebene Verwendung des Terminus "Prädikat" hat den Vorzug, daß mit sie allen Beschreibungsmodellen korrespondiert, denen die konstituentenstrukturgrammatik oder die genera- 
tive Transformationsgrammatik zugrunde liegen. Denn der Terminus "Subjekt" entspräche jeweils der Nominalphrase Np auf der 1. Verzweigungsstufe, während der Terminus "Prädikat" der Verbalphrase Vp entspräche, zu deren Bestandteilen weitere Nominalphrasen gehören, die in der Terminologie der traditionellen Grammatik als "Objekte" und als "Adverbialbestimmungen" erfaBt werden.

Auch die Beschreibungsmodelle, die den Terminus "Prädikat" im Sinne von 'finites Verb eines Satzes' benutzen, haben gelegentlich die Notwendigkeit gesehen, einen oberbegriff für die satzglieder Prädikat, Objekte und Adverbialbestimmungen zu haben. Dieser Notwendigkeit entstammt der Terminus "Prädikatsverband" der Duden-Grammatik. ${ }^{7}$ Als definierter Begriff erfüllt er zwar seine Aufgabe, dennoch ist er kein sprechender Begriff, da sich aufgrund des Bestimmungswortes "Prädikat(s)-" und des Grundwortes "-verband" seine Bedeutung nicht schnell und klar ermitteln läßt. Er bedeutet offensichtlich nicht 'Verband des Prädikats' oder 'Prädikat als Verband', sondern wohl 'Verband von satzgliedern, die bei dem Prädikat, dem finiten Verb des satzes, stehen'.

Neben den beiden dargestellten Beschreibungsmodellen, die sich des Terminus "Prädikat", wenngleich mit unterschiedlichem Denotat, bedienen, gibt es noch ein drittes Beschreibungsmodell, das sich an dem Deskriptor für das finite Verb eines satzes erkennen 1äBt: Der Terminus "Satzkern" gehört zu einem Beschreibungsmodell, das auf der Dependenzgrammatik beruht. Er zeigt an, daB alle verschiebbaren satzglieder innerhalb eines Satzes, also auch das subjekt der traditionellen Grammatik, von dem verb des Satzes abhängen.

Angesichts der Tatsache, daB es sowohl in der sprachwissenschaft als damit auch in der schulgrammatik mehrere recht unterschiedliche Beschreibungsmodelle gibt, müBte sowohl in der sprachwissenschaft als auch in der schulgrammatik gefragt werden, durch welches Beschreibungsmodell die Satzstruktur des Deutschen und die anderer indogermanischer sprachen sowie die Relationen der satzglieder am klarsten verstehbar und durchschaubar gemacht werden. 
Da aber sicher jedes Beschreibungsmodell vorzüge und Nachteile aufweist, dürfte es kaum möglich sein, daß ein Gremium von Sprachwissenschaftlern eine Empfehlung zugunsten des einen oder anderen Beschreibungsmodells gibt, wohl auch nicht unter der Voraussetzung, daB mit einer solchen Empfehlung der schule und den Schülern nur ein gewisses Maß an sprachanalytischen Grundkenntnissen und Grundbegriffen ermöglicht werden soll.

Daher wäre zu prüfen, ob nicht zumindest die Fachdidaktiker der einzelnen schulsprachen in Kenntnis der vorhandenen Beschreibungsmodelle und in Kenntnis der Bedürfnisse der schüler und der Schule eine solche Empfehlung geben könnten. Da aber auch letzteres nur schwer realisierbar ist, bleibt als Ausweg aus dem kritisierten Terminuswirrwarr zum gegenwärtigen zeitpunkt wohl nur die Möglichkeit, in der Auseinandersetzung mit der KMK-Liste eine gewisse Vereinheitlichung in der satzgrammatischen Terminologie zu versuchen. Daher soll an dieser stelle der Versuch gemacht werden, für die satzgrammatischen Phänomene, die in Schulgrammatiken mit jeweils unterschiedlichen Termini bezeichnet werden, Vorschläge zu ihrer einheitlichen Bezeichnung zu machen.

Geht man davon aus, daB die KMK-Liste in der satzlehre keine Termini aus dem Beschreibungsmodell der Dependenzgrammatik vorschlägt, stellt sich im Blick auf die dargestellte unterschiedliche Verwendung des Terminus "Prädikat" die Frage, welches der beiden Beschreibungsmodelle, die mit den Termini "Subjekt" und "Prädikat" arbeiten, einer vereinheitlichten schulgrammatik zugrunde gelegt werden könnte.

Da es durchaus wünschenswert ist, daß das Beschreibungsmodell einer Schulgrammatik einem wissenschaftlich vertretbaren und in hohem Maße konsensfähigen Beschreibungsmodell entspricht, empfiehlt es sich, das Beschreibungsmodell zu wählen, das der Konstituentenstrukturgrammatik und der generativen Transformationsgrammatik sowie deren graphischen Darstellungen am meisten entspricht. Dies aber ist das Beschreibungsmodell, in dem der Terminus "Prädikat" mit dem Denotat 'Satzaussage, die alle verschiebbaren satzglieder mit Ausnahme des Subjekts umfaßt' verwendet wird. 
Eine Entscheidung für dieses Modell hätte folgende Vorzüge:

- Die Termini "Subjekt" und "Prädikat" bezeichneten die Makrosegmente, die Basisglieder von Sätzen sowohl des Deutschen als auch anderer indogermanischer sprachen.

- Diese Begriffe wären in ihrem Denotat identisch mit den Begriffen "Subjekt" und "Prädikat", wie sie in der Logik verwendet werden. Das Prädikat des satzes Er liebt Märchen ist nämlich nicht liebt, 'ist ein Liebender', sondern 'liebt Märchen', 'ist ein Märchenliebender', 'ein Märchenliebhaber'.

- Damit wären auch die Termini "Satzgegenstand" und "Satzaussage" adäquate Bezeichnungsvarianten für die Termini "Subjekt" und "Prädikat".

- Für eine Detailanalyse der Makrosegmente ließen sich systemkonforme Unterbegriffe definieren, so die Termini: "einfaches subjekt" fïr ein subjekt, das nur aus einem einzigen wort besteht: I $n g e$ schläft, $s i e$ schläft; "Subjektgruppe" für ein subjekt, das aus mehr als einem wort besteht: $D i e$ $M u t t e r$ schläft; "Subjektkern" für das Nomen oder Pronomen innerhalb einer subjektgruppe, das die notwendige Referenz-bzw. Konnexionsanweisung enthält:

$$
\begin{array}{r}
\text { Die } \quad \text { schläft. } \\
\text { Die Mutter schläft. } \\
\text { Sie schläft. }
\end{array}
$$

Der Subjektkern stellte auch das Bezugswort für Attribute dar, die in einer subjektgruppe vorkommen: Die $M u t t^{b}$ meines Freundes schläft.

- Im Blick auf das Makrosegment "Prädikat" lieben sich folgende systemkonforme Unterbegriffe definieren: "einfaches Prädikat" für ein Prädikat, das nur aus einer finiten verbform besteht: Der Vater $l i$ e $s$; "Prädikatsgruppe" für ein Prädikat, das aus mehreren verschiebbaren satzgliedern besteht:

$$
\begin{aligned}
& \text { Der Vater } l i e s t e i n e n \text { s } \quad \text { \& } m \text { a } n \text {. } \\
& E \text { i } n \text { e } n \quad R \circ m \text { a } n \quad l i \text { e } t \text { der Vater. }
\end{aligned}
$$

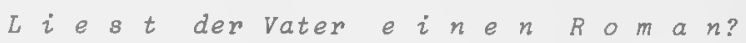


"Prädikatskern" für das finite verb innerhalb einer Prädikatsgruppe: - $\tau i e s t$ einen Roman; "Prädikatsteil" für die Teile einer prädikatsgruppe, die neben dem prädikatskern erscheinen, also die verschiedenen objekte und Adverbialbestimmungen. Diese Terminologie impliziert, daß in sätzen mit einem einfachen Prädikat das Prädikat gleich dem Prädikatskern ist: $E_{r} \quad l \quad i$ e s $t$.

Zu den genannten Unterbegriffen paßt auch systemkonform der Terminus "Prädikatsnomen", dessen Denotat allerdings wie folgt definiert sein sollte: 'Nomen, das im Prädikat vorkommt und durch eine finite Form der Verben sein oder werden als Kopula mit dem subjekt verknüpt ist'.

Beispiel: Er ist $L$ e $h r e r$. Er wird $L$ e $h r e r$.

Wegen der vorgeschlagenen Beschränkung des Terminus "Nomen" auf das Denotat 'Substantiv', 'Hauptwort' sollte für den Fall, daB ein Adjektiv durch eine finite Form der verben sein oder werden als Kopula mit dem subjekt verknüpft ist, der Terminus "Prädikatsadjektiv" verwendet werden.

Wie hoch der Grad der Entsprechung zwischen dem Beschreibungsmodell, das sich in den Baumgraphen der Konstituentenstrukturgrammatik und der generativen Transformationsgrammatik spiegelt, und dem hier vorgeschlagenen Beschreibungsmodell ist, zeigt die folgende Graphik: 

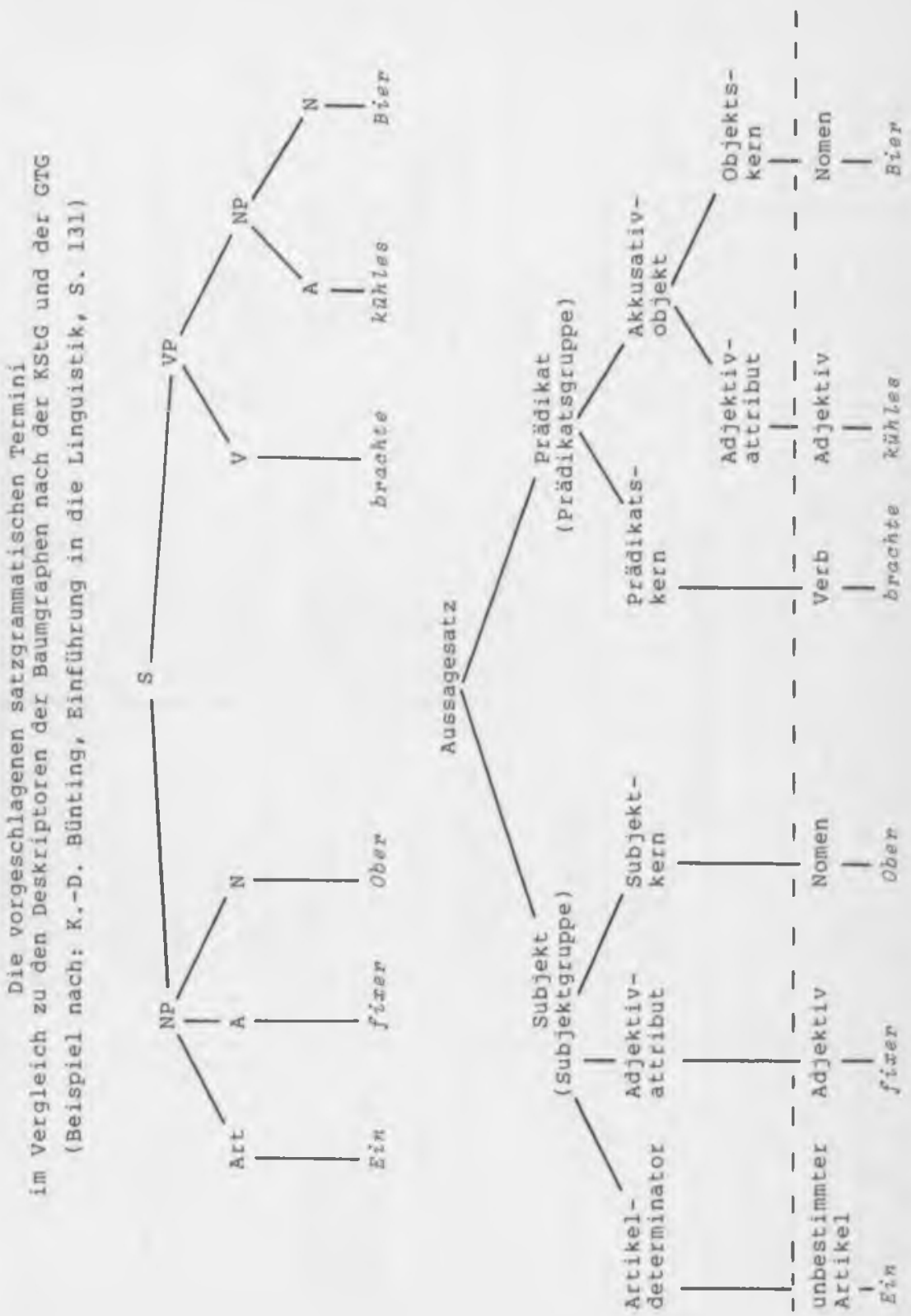


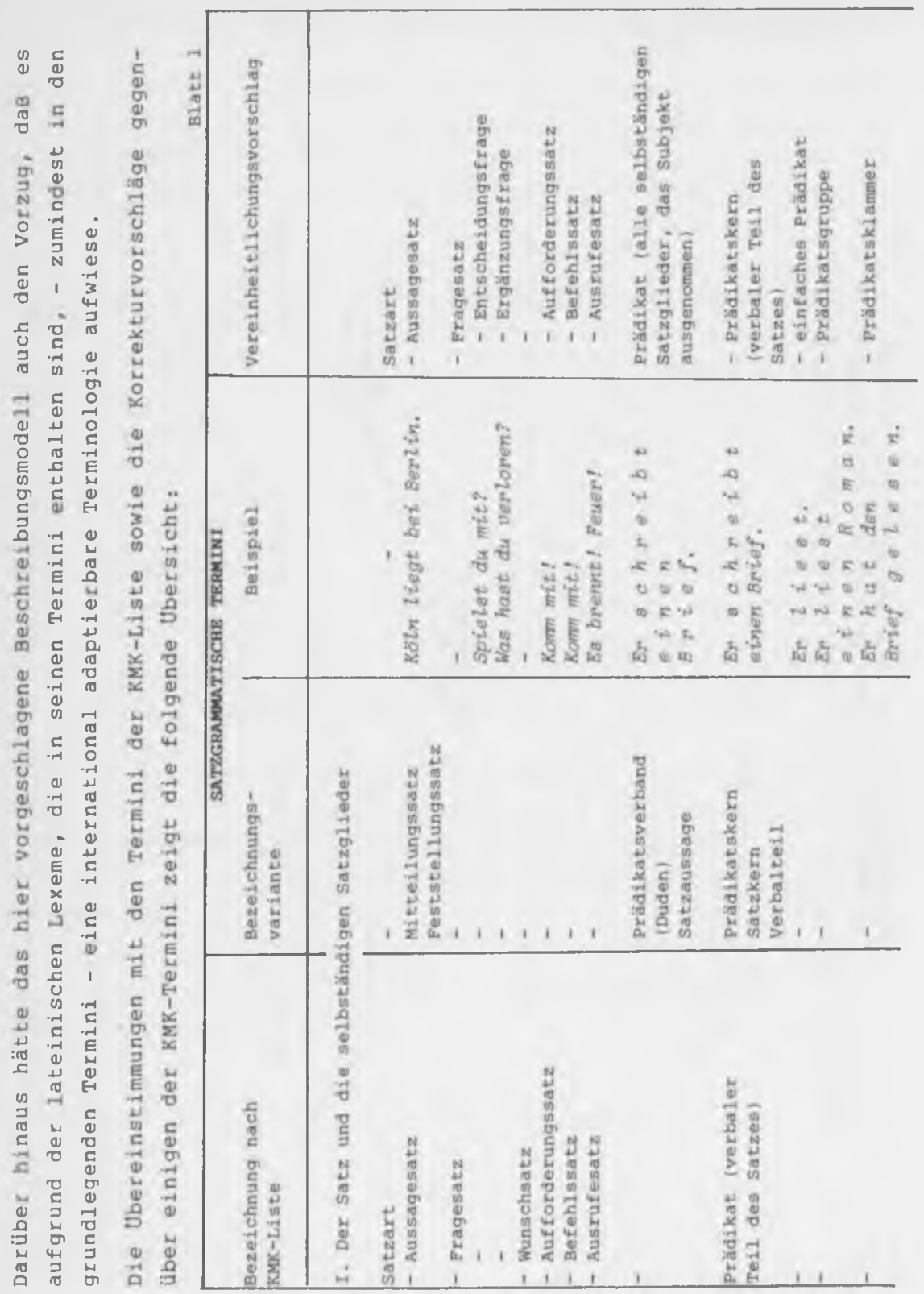




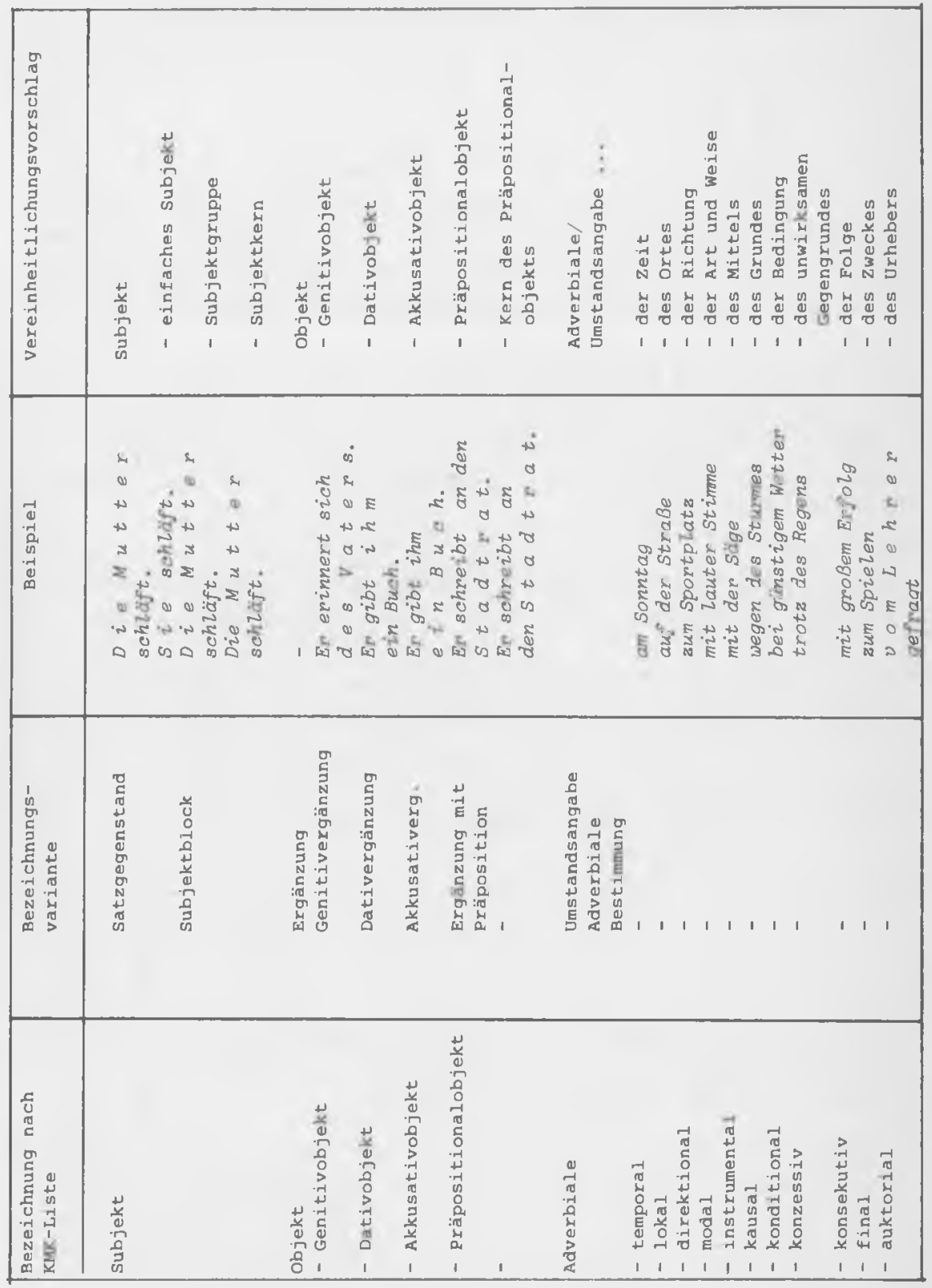


Ein weiteres terminologisches problem im Bereich der satzgrammatik stellt der Terminus "Hauptsatz" dar, der als Oppositionsbegriff $\mathrm{zu}$ dem Terminus "Nebensatz" relativ verständich war.

Da sich aber statt des Terminus "Nebensatz" aus guten Gründen immer mehr der Terminus "Gliedsatz" durchgesetzt hat, fehlt zumindest in der schulgrammatik eine Bezeichnung für den Teil eines Satzes, in den der Gliedsatz integriert wird. Als mögliche Termini böten sich an: "Matrixsatz" oder "Trägersatz".

Im Blick auf die Arten der Gliedsätze wäre zu empfehlen, daB die KMK-Liste neben den Termini "Subjektsatz" und "Objektsatz" auch Termini für die verschiedenen Adverbialsätze - in Analogie zu den Adverbialbestimmungen - aufnähme: "Temporalsatz", "Kausalsatz", "Modalsatz", etc.

Die Bezeichnungen für die Adverbialsätze im einzelnen zeigt die folgende Ubersicht auf der nächsten und übernächsten Seite.

Abschließend sei auf ein weiteres problem der Satzgrammatik hingewiesen, das einer Lösung bedarf: Durch welche Termini kann die syntaktische Leistung der Wortarten Artikel, Numerale, Demonstrativpronomen, Possessivpronomen, Interrogativpronomen sowie der Negation kein erfaßt werden? Hier wäre zu klären, ob diese Wortarten in Verbindung mit Nomina, syntaktisch gesehen, eine attributive oder eine determinatorische Funktion haben. Ist $\mathbf{z} . \mathbf{B}$. das wort $z$ wei in dem Satz 2 wei Kinder liefen über die straße ein Numeral a $t \quad r$ b $u t$ oder ist es ein Numeral d e $t$ e $r m i$ n a $t$ o r ?

Wenn man davon ausgeht, daß Attribute Anlagerungen an nominale Satzgliedkerne darstellen, dann kann die Anlagerung zwei als ein Numeralattribut interpretiert werden.

Andererseits kann die Funktion des wortes awei jedoch auch in Analogie zur Funktion des bestimmten und unbestimmten Artikels gesehen werden, so daß man besser von einem Determinator der bestimmten Menge, einem Numeraldeterminator spräche. 


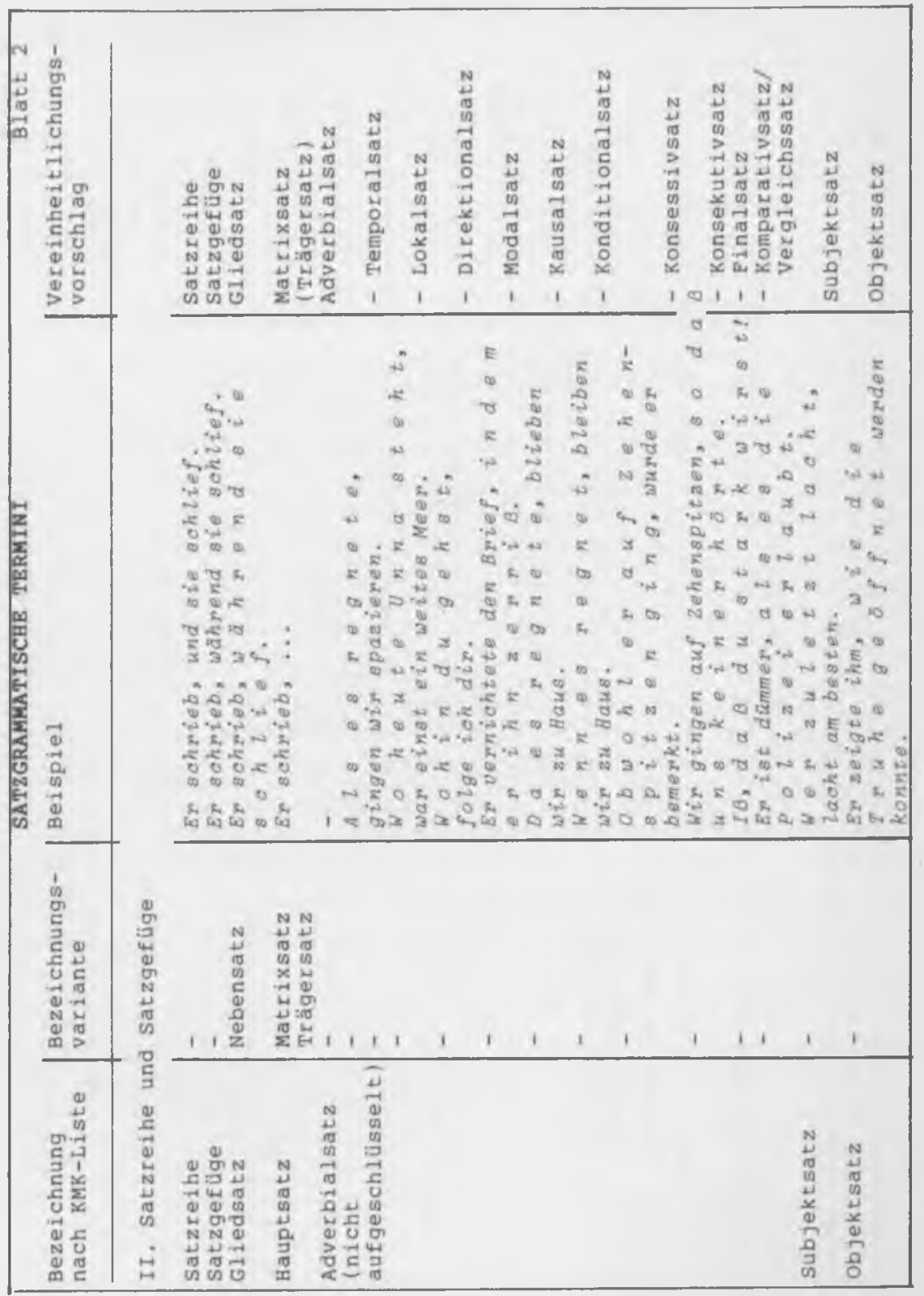




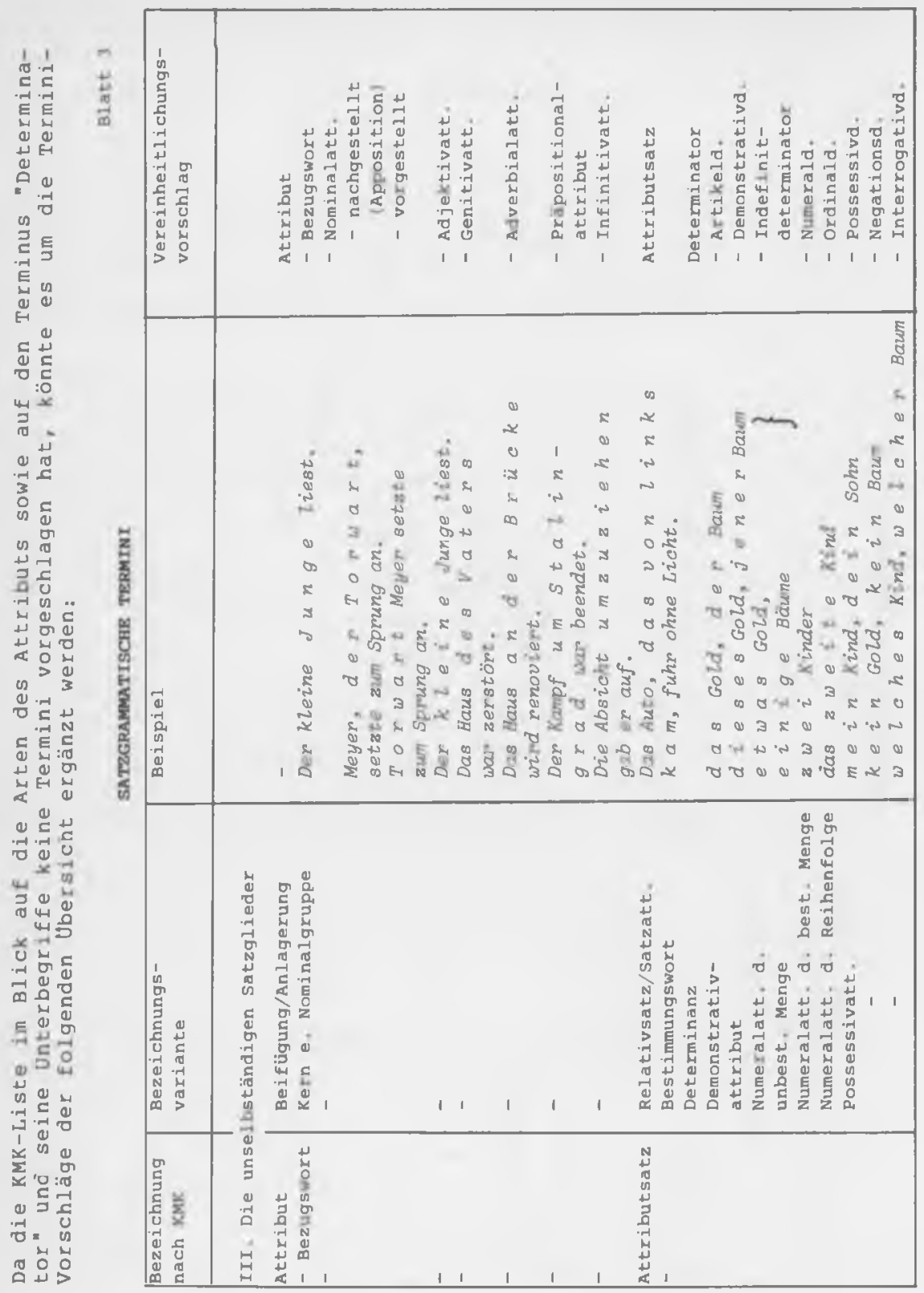




\section{Anmerkungen}

1 Dem Vergleich lagen folgende Sprachbuchreihen zugrunde:

1. Sprachbuch (ab 1970), Verlag Klett, Stuttgart, Bd. 5-7.

2. Sprachschlíssel (ab 1982), Verlag Klett, stuttgart, Bd. 5 und 6.

3. Lesen - Darstellen - Begreifen (ab 1975), Ausgabe A, Verlag Hirschgraben, Frankfurt, Bd. 5-7.

4. Wort und Sinn (ab 1980), Verlag Schöningh, Paderborn, Bd. 5-7.

5. praxis sprache (ab 1978), Verlag Westermann, Braunschweig, Bd. 6 .

6. Sprachbuch (Neubearbeitung), (ab 1978), Verlag Westermann, Braunschweig, Bd. 6 .

7. Verstehen und Gestalten (ab 1980), Verlag Oldenbourg, München, Bd. 1-3.

2 Vgl. dazu: Basisliste grammatischer Fachausdrücke, Arbeitskreis "Abstimmung der grammatischen Terminologie", Landesstelle für Erziehung und Unterricht, Stuttgart 1979.

3 Diese Ansicht beruht offensichtlich auf der Auffassung des stuttgarter Arbeitskreises, der mit dem Hinweis auf die Deklinierbarkeit der vier Wortarten Substantiv, Pronomen, Adjektiv und Numerale fúr die Beibehaltung des Terminus "Nomen" als Oberbegriff eintritt (Basisliste, S. 17).

4 So: Sprachbuch (Neubearbeitung), Verlag Westermann, Bd. 6.

5 So: Sprachschlissel, Verlag klett;

praxis sprache, Verlag Westermann;

Sprachbuch (Neubearbeitung), Verlag Westermann.

6 So: Sprachbuch, Verlag Klett;

Wort und Sinn, Verlag Schöningh;

W. Eichler U. K.-D. Bünting: Schulgrammatik der deutschen Gegenwartssprache, Verlag Schroedel, Hannover 1978.

Daß es zu dieser, wie der stuttgarter Arbeitskreis sagt "modernen Reduktion" (Basisliste, S. 4, Fußnote) gekommen ist, dürfe weitgehend darauf zuríckzuführen sein, daß sowohl Altsprachler als auch Neusprachler häufig anhand einfach gebauter sätze wie Comelia vocat oder Die Rose blüht in den prädikatsbegriff einfuhren.

7 Der Große Duden, Bd. 4, Grammatik, Mannheim 1973, S. 492; Lesen - Darstellen - Begreifen (neu), Verlag Hirschqraben, Frankfurt, 1982.

8 So: Wort und Sinn, Verlag Schöningh, Bd. 6 (1981), S. 107.

\section{Literatur}

Basisliste (1979): Basisliste grammatischer Fachausdrücke, Arbeitskreis "Abstimmung der grammatischen Terminologie". Landesstelle für Erziehung und Unterricht, stuttgart 1979.

Büting, Karl-Dieter (1972): Einführung in die Linguistik. Frankfurt 1972. Duden (1973): Der Große Duden, Bd. 4, Grammatik, Mannheim 1973, 3. neu bearbeitete und erweiterte Aufl.

KMK (1982): Verzeichnis grundlegender grammatischer Fachausdrücke, sekretariat der ständigen Konferenz der Kultusminister der Länder, Anlage VI z. NS. AK., 26.2.1982, Bonn. 